\title{
A Machine Learning Approach for Removal of JPEG Compression Artifacts: A Survey
}

\author{
Anagha R. \\ IV year B.E., \\ Department of CSE, \\ BMS College of Engineering, \\ Bangalore
}

\author{
Kavya B. \\ IV year B.E., \\ Department of CSE, \\ BMS College of Engineering, \\ Bangalore
}

\author{
Namratha M. \\ Assistant Professor, \\ Department of CSE, \\ BMS College of Engineering, \\ Bangalore
}

\author{
Chandralekha Singasani \\ IV year B.E., \\ Department of CSE, \\ BMS College of Engineering, \\ Bangalore
}

\author{
Hamsa J. \\ IV year B.E., \\ Department of CSE, \\ BMS College of Engineering, \\ Bangalore
}

\begin{abstract}
JPEG is a widely used image compression method. Though it is very efficient, it introduces certain artifacts and quantization noise. This paper is a detailed survey about various existing methods for the reduction of these artifacts. The paper explains each method and their advantages and drawbacks. Some of the methods mentioned are Weiner filtering, Image Optimization, Zero-masking, Local Edge regeneration, Multiple dictionary learning, Hybrid Filtering, Fuzzy filtering, Total Variation Regularization, Offset and Shift Technique, Post-processing et al. Also, a comparative study is made as to which method is suitable for which scenario.
\end{abstract}

\section{General Terms}

Image Processing, JPEG Image Compression, Artifact Removal, Image Noise reduction.

\section{Keywords}

Machine Learning, Feed - Forward neural networks, Blocking artifacts, Ringing artifacts, Blurring.

\section{INTRODUCTION}

Processing of images using mathematical operations by using any form of signal processing is Image Processing. The input to an Image Processing System is an image, such as a photograph or video frame. The output of image processing can either be a set of characteristics or parameters related to the input image or an image itself. Image Processing has a wide range of applications which include Image sharpening and restoration, Transmission and encoding, Machine/Robot vision, Color processing, Pattern recognition, Video processing, Microscopic imaging, in various fields like Medicine, remote sensing and others.

Image Processing is of two types: Digital Image Processing and Analog Image Processing. Digital Image Processing is the use of computer algorithms to perform image processing on digital images. Analog Image Processing is any image processing task conducted on two-dimensional analog signals using analog techniques. Digital Image Processing has numerous advantages over its analog counterpart. By changing the image format and resolution, it can be used in a wide range of applications. It is cost-effective and faster. The results of Digital Image Processing can also be viewed immediately.
Digital Image Compression is commonly used in Commercial Applications for Storage and Transmission of Digital Images when the Availability of Resources is limited. Compression of such data substantially affects the utilization of bandwidths over multimedia and IP networking Infrastructures. When the bandwidth availability is limited, the digital image to be transmitted over a network has to be compressed. Image Compression can either be Lossy or Lossless. During compression of an image, if all original data can be recovered when the image is decompressed, it is called Lossless Compression. On the other hand, if only part of the original data can be recovered during decompression due to elimination of certain information during the compression process, it is called Lossy compression. Many Methods exist for the compression of digital Images. JPEG (Joint Photographic Expert Group) is one of the most reliable and popular Image Compression methods that exist.

JPEG compression is a Lossy Compression method based on quantization of the Discrete Cosine Transform (DCT) coefficients of each 8 by 8 non-overlapping block of pixels that tile the image completely with respect to a quantization table followed by a lossless entropy encoder. It is used for Grayscale and Quality - Color images. The process of JPEG Compression consists of 3 sub-processes namely Discrete Cosine Transform (DCT), Quantization and Encoding (Compression).

The DCT Phase is a complex process which converts a snapshot of a real Image into a matrix of corresponding values. The Quantization Phase converts the values generated by DCT to simple number so that they occupy less bandwidth. This Phase of the JPEG Compression process is the Lossy phase. The compression process makes the quantized values as compact as possible. The Encoding or Compression Phase is usually lossless and uses standard compression techniques. The JPEG compression process is shown diagrammatically a follows.

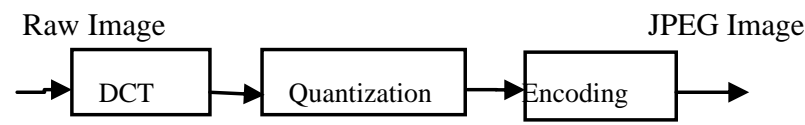

Fig. 1 JPEG Image Compression 
By scaling the elements of Quantization table as a function of a user-defined variable called Quality Factor $(Q)$, different compression ratios can be achieved. As the value of quality factor increases, more quantization noise is developed on the DCT coefficients, making the quantized coefficients values to get closer to each other [15]. As a result of this, the encoder can achieve higher compression ratios. However, this has a serious drawback. Coarse Quantization of the DCT coefficients may introduce certain visible artifacts in the image. These Compression Artifacts can be classified as follows.

- Blocking artifacts, which are mainly due to the coarse quantization of low-frequency DCT coefficients yielding decompressed image look like a mosaic at smooth regions.

- Ringing artifacts, which are mainly due to the coarse quantization of high-frequency DCT coefficients making the decompressed image exhibit noisy patterns known as ringing or mosquito noise near the edges. [15]

The reduction or removal of these compression artifacts has been of significant importance in order to enhance the visual quality of the resultant image. This has been the target of many researchers over the decades due to its importance in Image Processing. Several Methods have been proposed and implemented over the years for the removal of these compression artifacts. Some of them are Segmentation-based post-processing, Image enhancement by Space-Invariant filtering or Adaptive Spatial filtering, Image Restoration such as Projection Onto Convex Sets (POCS), Image Optimization, Wavelet-based Approach, Common Image De-blocking, Anisotropic Gaussian Filtering, One-Dimension oriented filtering, Hybrid Filtering, JPEG Reapplication et al.

The proposed method for removal of these compression artifacts involves Machine Learning. Machine learning is a branch of artificial intelligence that provides systems with the capability to learn without being explicitly programmed. It involves the development of computer programs that are selflearning, through which they grow and change when exposed to new data. The benefits of using Machine Learning algorithms for Image processing applications are that they can be applied to complex images or patterns. Since they are selfmodifying, they are also easier to use. They work better than predictive and heuristic approaches, resulting in higher accuracy.

This Literature Survey Paper intends to shed light on some of the recent techniques and methods proposed for the reduction of compression artifacts.

\section{LITERATURE SURVEY}

One of the methods of reducing the blocking artifacts is by using a wiener filter [1]. The wiener filters are used to decrease the blocking artifacts especially in the boundaries of the image segments. Since the images are divided into segments, each segment tends to be treated individually and provides different DCT coefficients for each block of the single image. This gives very coarse quantization values of the coefficients causing the original image and compressed image to look very different. The wiener filter here is used to produce very smooth images in such cases. Wiener filtering is applied to every single block of the image which is used to determine the wiener coefficients based on the amount of error in each block. The image de-blocking algorithm which makes use of this wiener filtering process consists of two steps. The First step involves providing an image to the wiener filter and to obtain the DCT coefficients. In the Second Step, quantization of the coefficients is performed by wiener filtering whose outputs are derived from the first step. The wiener filter gives a better output by improving the peak signal to noise ratio (PSNR) values of each block of the image. Hence it provides a better de-noised image than other methods.

In the Block-Wise Image Compression \&Reduced Blocking Artifacts Using Discrete Cosine Transform method [2], image compression is achieved through DCT and quantization methods. These values have been used for reducing blocking artifacts in reconstruction. Here the image space is mapped to a frequency domain. Now after applying DCT the next step is Quantization that reduces the redundancy of the data. At the receiver end the data is decoded, de-quantized and is reconstructed through inverse DCT. A DCT block is a group of pixels of an $8 \times 8$ window. The DCT grid is formed which are the horizontal and vertical lines that partition an image into blocks of images for the compression. The IDCT algorithm is used to generate reconstructed image. The 2-D DCT transform is been applied separately to each block. Irrelevancy reduction is then applied to the resulting transform coefficients of each block such that the relevant information is retained and the rest is removed.DCT is applied separately to each block of various sizes. After transformation the image is applied for quantization method and at the receiving end, decoding of the quantized DCT coefficients of each block is done separately, followed by the computation of the 2D-IDCT of each block. Later the decoder puts the blocks back together into a single image. It is recognized as equivalent to original image. This method produces good average PSNR values. It allows compression of images of different sizes as well as reduces artifacts.

Optimization method to reduce blocking artifacts [3] makes use of the image-gradient information of the JPEG images. The optimization method provides better results for low bit rate images. For every image an established equation is used. In this equation, the first part is used to retain the structural similarity between the images, and the remaining part of the equation brings out the smoothness at the borders of horizontal and vertical directions of the image. In order to avoid any loops when finding the gradient information, a closed form solution is obtained. In this solution the optimization formula is generated using a vector representation. Once the image to be transformed is represented by the vector optimization formula, the transition of the image takes place. In order to ensure that the transitions results in smooth images at the edges, guided filters are used. These filters are proven to be very effective edge-preserving smoothing filter. However the optimization solution in a closed form involves few implementation issues. This solution is not feasible for medium size images which make them computationally prohibitive. To overcome this drawback a sliding window computation approach is opted to deal with large matrices. In the sliding window approach optimization is done only for a defined size of the image irrespective of the input size of the image.

Using Zero-masking technique [4] on the DCT coefficients of some of the relocated blocks of the compressed image is another approach to reduce blocking artifacts in JPEG compressed Images. In the DCT domain, the vertical and horizontal boundaries of the adjoining blocks of the target block are perceptible. Next, a new block $(x, y)$ is created with row and column pixels. This process is done using a function which takes the primitive perceptible boundary values of the 
intermediate block as a parameter and these values are standardized using a two-dimensional step function. This Step-function employs Independent and Identical Distribution of noise resulting in zero mean value and less variance. On repeating this function for a few times, optimum results can be obtained. An advantage of this technique is that less number of repetitions is sufficient to minimize the blocking artifacts to a significant extent. It is also robust to noise and changes in illumination. However, it can result in slight alteration of the original image.

In JPEG artifact reduction via local edge regeneration [5], the authors propose to minimize not only blocking artifacts but also Blurring and Aliasing (Ringing) artifacts. Their method involves a two-stage process of enhancing the visual quality of JPEG Images. In the first step, blocking artifacts are eliminated. In the next step, blurring and aliasing artifacts are reduced. The removal of Blocking Artifacts is done by means of Boundary Smoothing and Guided Filtering. Aliasing Artifacts and blurring are reduced around the edges using local edge regeneration. This technique is quite effective at improving the visual quality of the image since it gives very good results in terms of PSNR and SSIM scores. However it is not quite effective for JPEG images compressed at extremely low bit rates i.e., $<0.15 \mathrm{bpp}$, like most methods. But these drawbacks can be overcome using better edge detection algorithms or by having some prior information about the edges of the images. This technique also does not consider the artifacts generated by quantization of DCT components of the chrominance channels and hence is not applicable to color artifacts.

In Reduction of blocking artifacts using multiple dictionary learning [6] method, a single dictionary of the values for different kinds of image segments is replaced by a set of small sub-dictionaries or subspaces each of which has values related to one kind image blocks. When the compressed image is divided into segments or blocks, each block is then compared to the subspace to check the similarity. If that texture matches with the data in subspace then that subspace (values) are chosen to represent that block of the image. This process is continued until all the blocks of the image get a subspace so that the final image is represented using those values. Unlike in the other methods, this method makes use of the subspaces to determine the texture of the image block rather than using the traditional DCT coefficient values.

The blocking artifacts removal using hybrid filtering method [7] involves a process where every image to be de-blocked is divided into segments which undergo filtering. This hybrid filtering method involves a two-step filtering process of the images. These steps are edge preserving and low pass filtering. This method makes use of the frequency information of the image to reconstruct the DCT coefficients based on their probability distribution, and also uses the spatial information to remove the block discontinuities and also to improve the line and edge quality of the image. The resultant images from both the processes are then combined in a careful manner to produce a better de-blocked image. The hybrid filtering involves an adaptive filtering technique, 1-Dimension filtering which is done along its linear structure of the image. Once the images are filtered, they undergo a careful combining process based on their gradient energy of the image segments. This method is designed to consider output images of textured regions from the edge adaptive filters and images for flat regions from low pass filters. This method overcomes some of the drawbacks that are caused during the 1-Dimension filtering. For example, the edge adaptive filter does not filter the flat regions and low pass filter smoothes the images. However the hybrid method also exhibits drawbacks like it cannot remove the noise near the lines and edges in an image. This problem is solved by introducing a pre-filter to the image before finding out the weighted average of the image segments in order to result in sharp images with noise reduced to great extent.

In artifact removal using fuzzy filtering method [8], a combination of fuzzy filtering and the powerful anisotropic diffusion equations is employed. It is known that the selection of adaptive threshold parameter that forms a diffusion coefficient has also improved performance of the algorithm. This technique was developed based on directional nature of blocking artifacts and is found to be efficient over any other directional and fuzzy filter methods. Anisotropic diffusion is a method by which reduction of artifacts is achieved without removing any essential parts of the image contents such as edges, lines and other details which are meant to be important for interpretation of image. Firstly, fuzzy filtering is applied to those block of pixels that have been suffering from blocking artifacts, then the directional anisotropic diffusion is applied so as to improve the quality of the image and finally adaptive 2Dfuzzy filtering is applied to the pixels that suffer from ringing artifacts. Since the direction and activity of pixels have been taken into consideration, this method has overcome many limitations of the conventional non-linear filters .It is known that the method has improved the visual quality of compressed images which were measured by the PSNR and MSSIM parameters.

The Blocking Artifacts Reduction in Compressed Data using edge Identification method [9] has two major steps. The first step involves edge identification in compressed data and second step is a compound method that is carried out to reduce blocking artifacts. Since all the computations are meant to be performed within the DCT domain, the original image is not affected. The proposed method gives better visual effects under both low bit rate encoding and high bit rate encoding scenarios. There exist three key parameters that need to be identified. They are edge orientation, edge strength and edge offset. Since this method assumes that noise has a Gaussian distribution, these facts lead to good separation between signal and noise. The proposed algorithm beats some of the classic methods given the result of edge block identification and also blocking artifact reduction.

In Dictionary Learning and Total Variation Regularization Method [10], decompression of image is done in order to produce an image with less artifacts and better texture. This proposed method has four phases namely image compression, dictionary learning, TV method and jpeg dithering. The first step in image compression is DCT. The image is divided into $8 * 8$ blocks. Since DCT is designed to work with pixel images of range -128 to 127 , thereby the original image is leveled off by subtracting with 128 from each entry. Each element in DCT coefficients is multiplied with corresponding element in quantization matrix. This obtained result undergoes entropy encoding. Dictionary learning is a two-step iterative method. The first step is using OMP algorithm to update encoding coefficients. The second step is using SVD algorithm to update dictionary. The advantage of this model is that determination of sparse representation of the restored image in the learned dictionary so that restored image keeps the features of the original image is possible. In TV method they have used first order primal dual algorithm to update the restored image. To the obtained result dithering noise is added in the DCT domain to improve quality of image. From the 
experimental results it is observed that Dithering enhances the resultant image obtained from total variations which provide better PSNR and structural similarity.

The method of reducing blocking artifacts by post filtering [11] removes the coding artifacts in compressed medical images. The artifacts are removed by changing some of the DCT coefficient values in both the smooth and non-smooth regions of the blocks of the images. The input images are first categorized under a particular mode and then filtered. Thus a mode detection algorithm is introduced before the filtering of the image. Once the mode is detected, a less level computation capacity filter which is capable of handling three modes namely smooth, intermediate, and non-smooth modes is introduced for appropriate frequencies. This filter attempts to remove the artifacts in the blocks of the image with minimum loss of image data. Here only shifting of image blocks is done instead of dividing them, hence it will reduce the load for the filter for removal the existing artifacts. The type of filter to be used is decided by the particular block of the image i.e., smooth or intermediate or non-smooth. The identification of the mode of the block is done by examining the action of the image at the boundary of the image block i.e., considering the edges of the block. Once the mode is determined then appropriate filters are applied and the artifacts are removed.

The post processing approach for ringing artifact reduction method [12] consists of DCT domain filtering and image domain post processing that will reduce ringing artifacts on the resized block-DCT domain. This method generates mask map of the overshoot region. And combines ripple reduced image and an overshoot reduced image in accordance to the mask map that was generated in the image domain for obtaining ringing artifact reduced image. This method reduces ringing artifacts without further blurring, as compared to other previous approaches that had to find a compromise between reduction of blurring and ringing artifacts. It produces visually finer images than most of the previous ringing artifact reduction approaches. Image resizing is the most fundamental operation in today's world as the image must be displayed on various devices. Ringing artifacts are more dominant around textual images, while the blocking artifacts are more visible in natural images. It is meant that the textual parts and pictorial regions in the document are separated from each other by automatic segmentation. This method reduces artifacts by combining overshoot reduced images and ripple reduced images. The ringing artifact reduced image is constructed from two OR filtered images by performing the image-domain masking operation on those regions

Reduction of blocking artifacts by quality adaptive trained filters [13] makes use of the quality metrics of the image to be de-blocked. Here the non-compressed images are set as targets for the algorithm to achieve from the compressed images containing blocking artifacts. The adaptive filtering algorithm contains two steps. In the first step, each pixel of the block image is classified based on its boundary neighbors. An Adaptive Dynamic Range Coding classification method is applied here, which obtains the information and the complexity of the neighboring pixels and thus classifies the given pixel into a selected category or a class. Once the classification is done, all the pixels belonging to a specific category or class are grouped into one and their corresponding coefficients are measured and obtained using the Mean Square Error (MSE) method. These coefficients are now stored in the look up table for any further references. Once an input image is evaluated based on its quality metrics the algorithm searches for the most suitable coefficients values for the blocks of the image and based on these values of pixel classification, the image is further filtered.

Offset and Shift Technique [14] is one of post-processing algorithm. Post processing algorithms are used to eliminate blocking artifacts in a highly compressed data and improve the quality of images. To achieve de-blocking, original image is not required as reference. This is the main advantage of post-processing algorithms. Offset and shift technique is a deblocking filter with low computational complexity and has three filtering modes working in spatial domain. Mode decision procedure is decided based on the observation made on the pixel behavior across the boundary. The difference is computed and compared with threshold value. If the value is larger than the threshold value, then it belongs to a real edge. Hence the pixel vector is skipped. Otherwise, suitable mode will be chosen based on the activity of the pixel vector to filter it. Activity is the variation of the pixel vector. Based on the calculated activity, pixel vector is classified into one of the 3 regions namely smooth, intermediate and complex region. Filtering is done in each region. This technique yields better result by preserving the edge details, removing artifacts in the smooth areas. According to the simulation results, the proposed method outperforms other de-blocking algorithms with respect to image quality measures such as PSNR (Peak Signal-to-Noise Ratio) and SSIM (Structural SIMilarity).

\section{CONCLUSION}

In this paper, a comparative study of various artifact removal techniques was done. Weiner Filter gives better PSNR values for each image block. DCT method allows compression of images of different sizes along with the reduction of artifacts. Optimization method is effective for edge preservation; but in the closed form, it has a few implementation issues. Zero masking technique is robust to noise and changes in illumination, but it can result in slight alteration of the image. The local edge regeneration method improves the visual quality of images significantly; but it is not applicable for color artifacts. Unlike other methods, multiple dictionary learning method makes use of the subspaces to determine the texture of the image block rather than using the traditional DCT coefficient values. Although Hybrid filtering overcomes the drawbacks of 1D filtering, it cannot remove noise near lines and edges in the image without pre-filtering. Fuzzy filtering overcomes most of the drawbacks of conventional filters. Offset and Shift technique yields better edge preservation and PSNR values.

On Comparison of all the methods mentioned, Filtering methods are usually highly effective, but they suffer from High Computational Complexity. Some of these methods also require Input parameters. In most situations, Dictionary learning is efficient and computationally less-expensive.

\section{ACKNOWLDEGEMENTS}

The work reported in this paper is supported by the college through the TECHNICAL EDUCATION QUALITY IMPROVEMENT PROGRAMME [TEQIP-II] of the MHRD, Government of India. 


\section{REFERENCES}

[1] S.Gayathri Tejaswini, M. Ramalakshmi, M. Santhi, H. Rahul and Hemanth Nag, "Reduction of Blocking Artifacts of DCT Compressed Image Based on Block Wiener Filtering", International Journal of Advanced Research in Electronics and Communication Engineering (IJARECE) Volume 4, Issue 3, March 2015.

[2] Dr.S.S.Pandey, Manu Pratap Singh and Vikas Pandey, "Block wise image compression \& Reduced Blocks Artifacts Using Discrete Cosine Transform", International Journal of Scientific and Research Publications, Volume 5, Issue 3, March 2015.

[3] Reza Pourreza-Shahri, Siamak Yousefi and Nasser Kehtarnavaz, "Optimization method to reduce blocking artifacts in JPEG images", Journal of Electronic Imaging, November 2014.

[4] Jyothi Mishra, "Suppression of Blocking Artifacts in Compressed Images", Project Thesis, NIT Rourkela, May 2014.

[5] S. AlirezaGolestaneh and Damon M. Chandler, "Algorithm for JPEG artifact reduction via local edge regeneration", Journal of Electronic Imaging, January 2014.

[6] Yi Wang and Faith Porikli, "Multiple Dictionary Learning for Blocking Artifacts Reduction", IEEE International Conference on Acoustics, Speech and Signal Processing (ICASSP), 2012.

[7] Tuan Q. Pham and Lucas J. van Vliet, "Blocking artifacts removal by a hybrid filter method".

[8] EhsanNadernejad, SørenForchhammer, and JariKorhonen, "Artifact Reduction Of Compressed Images And Video Combining Adaptive Fuzzy Filtering And Directional Anisotropic Diffusion", 3rd European Workshop on Visual Information Processing (EUVIP), 2011.

[9] Fang Zhu, "Blocking Artifacts Reduction in Compressed Data",International Conference on Computer Engineering and Applications, 2009.

[10] Chitra PS, Niyas Ibrahim and Dr. A NeelaMadheshwari, "Artifacts Removal in JPEG Decompression via learned dictionary and Total variation regularization", International Conference on Current Techniques in Medical Image Analysis, 2014.

[11] Jagroop Singh, Sukhwinder Singh and Dilbag Singh, "Reduction of blocking artifacts by post filtering algorithm", Journal of Information and Computing Science,2013.
[12] Bobby Lukose and P. Roobini, "A Post Processing Approach for Ringing-Artifact Reduction", IJISET International Journal of Innovative Science, Engineering \& Technology, September 2014.

[13] Ling Shao, Jingnan Wang, IhorKirenko and Gerard de Haan, "Quality Adaptive Trained Filters For Compression Artifacts Removal”, IEEE 2008.

[14] YuvinderDandiwal, KirtiSachdeva, "The Study Of Various Approaches For Removal Of Blocking Artifacts In Spatial Domain", International Journal of Computer Science and Mobile Computing, Vol.2 Issue. 12, December 2013.

[15] BasakOztan, Amal Malik, Zhigang Fan and Reiner Eschbach, "Removal of Artifacts from JPEG Compressed Document Images", SPIE- IS\&T.

[16] M. Hanmandlu. J. See, S. Vasikarla, "Fuzzy Edge Detector Using Entropy Optimization", Proceedings of the International Conference on InformationTechnology: Coding and Computing, 2004.

[17] Y. Luo, R.K. Ward, "Removing the Blocking Artifacts of Block-Based DCT Compressed Images", IEEE Trans. Image Processing, 12, 2003.

[18] S. Singh, V. Kumar, H.K. Verma, "Reduction of blocking artifacts in JPEG Compressed Images", Digital Signal Processing, 17, 2007.

[19] J. Kim and Chun-Bo Sim, "Compression Artifacts Removal by Signal Adaptive Weighted Sum Technique", IEEE Transactions on Consumer Electronics, November 2011.

[20] Amir Z. Averbuch, A.Schclar and David L. Donoho, "Deblocking of block-transform compressed images using weighted sums of symmetrically aligned pixels", IEEE Trans. Image Process., 2005.

[21] Chun-Su park, J. Hyungkim and Sung-Jeako, "Fast Blind Measurement of Blocking Artifacts in both Pixel and DCT domain", Journal of Mathematical Imaging and Vision, 2007.

[22] Yen-Yu Chen, Ying-Wen Chang and Wen-Chien Yen, "Design a deblocking filter with three separate modes in DCT - based coding", Journal of Visual Communication and Image processing, 2008.

[23] G.Zhai, W.Znag, X-Yang and W.Lin, "Efficient image deblocking based on post filtering in shifted windows", IEEE Transactions Circuits Systems \& Video Technology, 2008. 